\title{
Relación entre los atributos de ensambles pleustónicos y la complejidad de hábitat generada por macrófitas en el río Paraná Medio
}

\author{
Facelli Fernández Florencia ${ }^{1,2}$, Zilli Florencia ${ }^{2}$ \\ ${ }^{1}$ Facultad de Humanidades y Ciencias (FHUC-UNL). Ciudad Universitaria, Pasaje el Pozo, \\ 3000, Santa Fe, Argentina. ${ }^{2}$ Instituto Nacional de Limnología (INALI UNL-CONICET). \\ Ciudad Universitaria, Pasaje el Pozo, 3000, Santa Fe, Argentina.
}

E-mail: florzeta1979@yahoo.com.ar

\begin{abstract}
RESUMEN. La complejidad de hábitats generada por macrófitas en ambientes acuáticos favorece la diversidad y producción de la biota. A pesar de la gran abundancia de macrófitas, existe escasa información en humedales aluviales del río Paraná. Nuestro objetivo fue determinar la relación de los atributos de ensambles de invertebrados pleustónicos con la complejidad de hábitat generada por los sustratos vegetales. Se recolectaron muestras en seis humedales en la llanura aluvial. Para determinar las medidas de complejidad de hábitat, se obtuvieron la biomasa, riqueza y dimensión fractal promedio de partes sumergidas de las macrófitas. Los invertebrados se identificaron y categorizaron en grupos funcionales, se contabilizaron y se obtuvo su biomasa por pesaje directo o empleando factores de corrección disponibles. Los atributos se relacionaron con la complejidad de hábitat empleando correlaciones lineales. Se registró una elevada diversidad, densidad y biomasa de taxones y de diferentes grupos funcionales de macroinvertebrados. Hubo una relación directa de la complejidad de hábitat con la riqueza y densidad del grupo de mayor especificidad por el sustrato vegetal (trituradores, herbívoros, raspadores) e indirecta con la densidad total y de recolectores. Concluimos que las macrófitas son generadoras de hábitat para diversos y abundantes macroinvertebrados en el río Paraná. Los atributos se relacionan con la complejidad de las partes sumergidas diferencialmente de acuerdo con su especificidad por el sustrato. Las macrófitas y los invertebrados pleustónicos intervienen por deriva en diferentes procesos local y regionalmente, por lo que aportarían a la resiliencia del sistema río-llanura aluvial.
\end{abstract}

Palabras clave: Complejidad, diversidad, pleuston, resiliencia.

ABSTRACT. The habitat complexity generated by macrophytes favours biota diversity and productivity. However, little is known in floodplain wetlands of the Paraná River. Our objective was to evaluate the relationship between pleuston attributes and habitat complexity generated by macrophytes. Samples were collected in six floodplain wetlands. Submerged plant biomass, richness and the fractal dimension were obtained to determine different measurements of complexity. Invertebrates were identified and classified according to different functional groups. The organisms were counted and their biomass was determined by direct weight or using available factors. Correlation tests between the attributes and habitat complexity were performed. A high diversity, density and biomass of taxa and functional groups were found. Complexity was directly related to richness and density of invertebrates with high affinity to plant substrate (shredders, scrapers, herbivorous) and indirectly to overall and collector density. Macrophytes provide habitat for diverse and abundant macroinvertebrates in the Paraná River. Attributes are related to the complexity of the submerged portions of macrophytes differentially in relation to substrate-affinity of macroinvertebrates. Both, macrophytes and invertebrates participate by drift in local and regional processes, thus supporting floodplain-river system resilience.

Keywords: Complexity, diversity, pleuston, resilience. 


\section{INTRODUCCIÓN}

La complejidad estructural de hábitats favorece la diversidad taxonómica y funcional (Arrington et al. 2005; Kelaher, 2003; MacArthur \& MacArthur, 1961; Mormul et al., 2011; Ricklefs \& Lovette, 1999). Las macrófitas cumplen un papel importante en la estructuración del hábitat (Giorgi et al., 2005), no sólo porque al disminuir la velocidad de la corriente facilitan la deposición de sedimentos (Sand-Jensen, 1998), sino porque brindan una superficie y subdivisión de hábitat para los invertebrados. Debido a esto, representan un elemento del paisaje fundamental a tener en cuenta para analizar la relación entre complejidad espacial y atributos de los ensambles de invertebrados asociados o pleustónicos (McAbendroth et al., 2005; Thomaz et al., 2008; Padial et al., 2009). En particular, los métodos basados en fractales proveen índices que miden la heterogeneidad espacial (Saravia et al., 2012) y son ampliamente utilizados en ecología (Li 2000; Brown et al., 2002). Teniendo en cuenta que una dimensión fractal mayor representa formas de hojas y tallos más finamente divididas y diseccionadas y una menor tiene formas más simples (Dibble \& Thomaz, 2009), la complejidad estructural de las raíces de diferentes especies de plantas acuáticas puede analizarse a través de su dimensión fractal.

Debido a su variada arquitectura, las especies de plantas acuáticas pueden sostener ensambles de invertebrados con diferente biomasa, tamaño y composición taxonómica y funcional (Cyr \& Downing, 1988; Cheruvelil et al., 2002; Dvořak \& Best, 1982; Feldman, 2001; Hanson, 1990). En los humedales de la llanura en el río Paraná Medio las macrófitas presentan una gran diversidad taxonómica y ecológica (Franceschi et al., 1985; Neiff et al., 2014; Schneider et al., 2015), y contribuyen con una gran biomasa al sistema (Sabattini \& Lallana, 2007). Así mismo, las plantas acuáticas constituyen un sustrato para bacterias y algas del perifiton, y son utilizadas por muchos invertebrados y vertebrados como hábitat, para la alimentación, refugio, reproducción, etc. (Agostinho et al., 2003; Neiff y Poi de Neiff, 2003). A pesar de su importancia, las macrófitas en humedales están amenazadas globalmente, lo que representa un riesgo para la conservación tanto de las plantas acuáticas como de los ecosistemas que sostienen (O'Hare et al., 2018; Zhang et al., 2017).

Muchos estudios se han desarrollado en el río Paraná en su tramo argentino-brasilero acerca de las macrófitas como hábitat para macroinvertebrados (Higuti et al., 2007; Paporello de Amsler, 1987; Poi de Neiff y Neiff, 2006; Souza Franco \& Takeda 2000; Thomaz et al., 2008). Sin embargo, es aún poco conocido el efecto que la complejidad de hábitats generada por las macrófitas tiene sobre la densidad, riqueza, diversidad y biomasa de invertebrados pleustónicos en humedales aluviales del río Paraná Medio. El objetivo de nuestro trabajo fue determinar la relación entre los atributos de los ensambles de invertebrados pleustónicos y la complejidad de hábitat generada por los sustratos vegetales. Hipotetizamos que existe una relación positiva entre los atributos del ensamble de invertebrados (la densidad, biomasa, diversidad y grupos funcionales tróficos) y la complejidad generada por las macrófitas. Predecimos que, los grupos funcionales tróficos asociados al consumo de macrófitas y perifiton (trituradores, herbívoros, raspadores) aumentan su proporción en relación al resto de los grupos funcionales tróficos (colectores y depredadores), al aumentar la complejidad de las macrófitas. Este estudio aporta información sobre los atributos de invertebrados pleustónicos (densidad, biomasa y riqueza de taxones y grupos funcionales) que habitan humedales del río Paraná Medio. Asimismo, se reporta por primera vez información sobre la dimensión fractal de especies vegetales y sobre complejidad estructural de las macrófitas para la región de estudio.

\section{MATERIALES Y MÉTODOS Área de estudio}

El río Paraná recorre 2570 km desde sus nacientes en Brasil hasta su desembocadura en el Río de la Plata, siendo el segundo río de mayor longitud largo de Sudamérica luego del río Amazonas. El área de estudio incluyó a la llanura de inundación del río Paraná medio 
en una sección transversal entre las ciudades de Santa Fe y Paraná (Figura 1). Los sitios de estudio se ubican dentro de la ecorregión Delta e islas del Paraná (Burkart et al., 1999), donde los cuerpos de agua se caracterizan por presentar parte o toda la zona litoral colonizada por macrófitas flotantes como Pontederia crassipes Mart., P. azurea Sw., Pistia stratiotes L., Salvinia spp. y Azolla spp.; sumergidas como de extracción y red de $200 \mu \mathrm{m}$ de abertura de malla. Se colectó un total de 30 muestras, es decir 5 replicas por humedal. Cada replica estuvo conformada en algunos casos por varias especies vegetales y otras por una sola especie, dependiendo de las características de cada humedal. Las mismas fueron trasladadas cuidadosamente al laboratorio en bolsas plásticas.

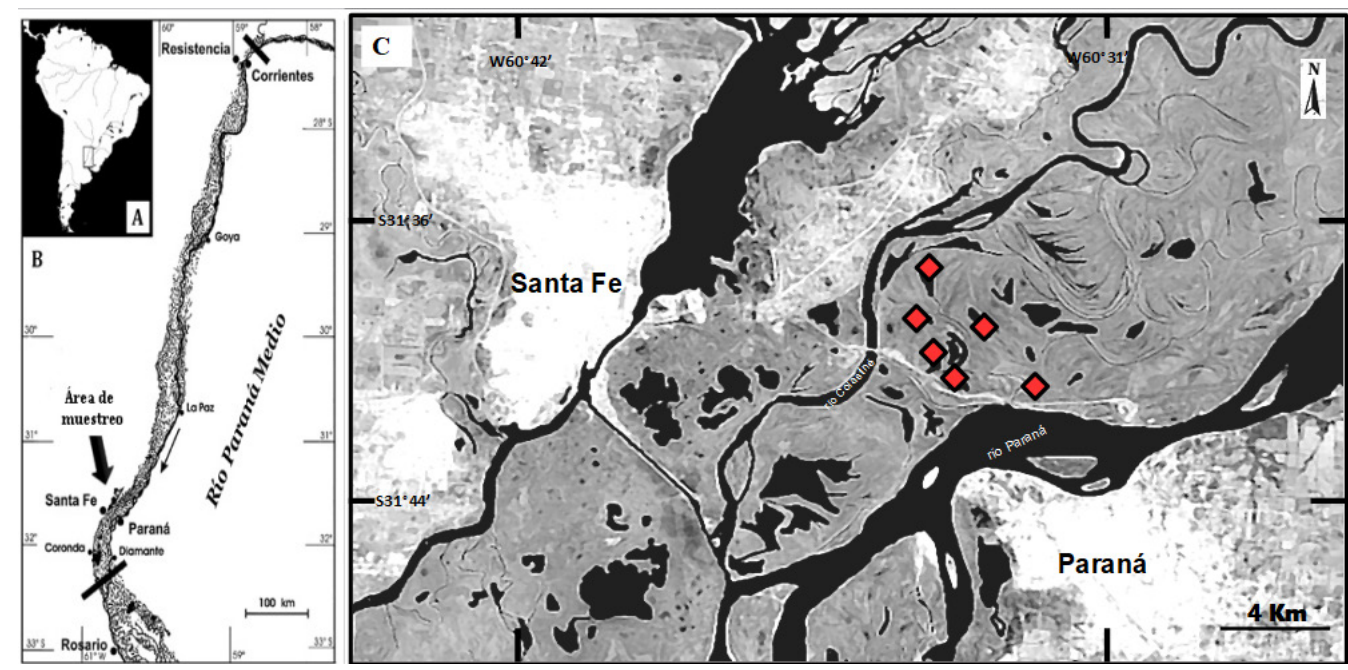

Figura 1. Ubicación del área de estudio. A. Sudamérica indicando la ubicación del río Paraná Medio; B. De la Ilanura aluvial y C. Localización de los sitios de muestreo (rombos rojos) (imagen satelital de la colección del INALI-CONICET-UNL).

Figure 1. Location of the study area. A. South America indicating the location of the Middle Paraná River; B. The floodplain and C. Location of the sampling sites (red diamonds) (satellite image of INALI-CONICET-UNL collection).

Utricularia spp., Ceratophyllum demersum L., Myriophyllum aquaticum (Vell.) Verdc. y Cabomba caroliniana A. Gray y por especies palustres como Schoenoplectus californicus (C. A. Mey.) Sojak, Polygonum punctatum Elliott. y Ludwigia peploides (Kunth.) P. H. Raven. El muestreo se realizó durante un período de aguas medias encauzadas (Octubre de 2014). Se escogieron seis humedales permanentes (Figura 1), los cuales difieren en origen, conexión al río, tamaño, distancia a los cauces mayores (río Paraná y río Colastiné).

\section{Muestreo y procesamiento de macrófitas}

En las zonas litorales se colectaron muestras de macrófitas con copo de $0,07 \mathrm{~m}^{2}$ de superficie
En el laboratorio, se lavaron las macrófitas con agua de red con un tamiz de $200 \mu \mathrm{m}$ de abertura de malla para retener los invertebrados adheridos a las raíces y partes vegetales sumergidas. Las macrófitas fueron identificadas según claves disponibles Burkart (1957,1969, 1974) Burkart y Bacigalupo (1979), Cabrera (1968) y Pott y Pott (2000). Se determinó la composición y biomasa en peso seco de las partes sumergidas de cada especie de macrófita, tras secarlas en estufa a $60^{\circ} \mathrm{C}$ por 72 horas y determinar su peso en balanza OHAUS de 0,01 mg de precisión.

Para analizar la complejidad fractal de las raíces y partes sumergidas, éstas se digitalizaron con escáner (HP Deskjet 2135). Las imágenes trabajadas tuvieron un tamaño de 1700×2200 
pixeles, se convirtieron a blanco y negro mediante la función treshold utilizando el programa Image 1.51KJava 1.6.0_24. Finalmente, se utilizó el Plugin FracLac del programa ImageJ $1.51 \mathrm{~K}$ para obtener la dimensión fractal aplicando boxcounting method, empleando en todos los casos un tamaño de cuadrado de 20 entre 10 y 110 pixeles $(0,45$ y $5 \mathrm{~mm})$. Se determinó la dimensión fractal de las raíces y partes sumergidas de cinco ejemplares de cada especie vegetal de todas las especies colectadas, para luego obtener la dimensión fractal promedio por especie (D). Se seleccionaron cinco ejemplares de cada especie, para asegurar que, con este número, se obtuviera un promedio representativo que abarcara una muestra de la variación intraespecífica.

\section{Procesamiento de invertebrados}

Se identificó y contabilizó el total de los organismos bajo lupa estereoscópica y/o microscopio óptico, empleando claves taxonómicas disponibles hasta el nivel de familia o subfamilia (Domínguez y Fernández, 2009; Merritt \& Cummins, 1996). Los oligoquetos se identificaron hasta el nivel de especie (Brinkhurst y Marchese, 1989), debido a su amplia distribución en la llanura aluvial del río Paraná Medio y a su frecuencia de ocurrencia. Los invertebrados fueron asignados a grupos funcionales tróficos de acuerdo a Cummins et al. (2005), estudios locales de dieta (Galizzi et al., 2012) y observaciones personales. Los invertebrados se agruparon en relación con el grupo funcional y dependencia de la fuente de alimento como: Raspadores, trituradores y herbívoros (RTH), recolectores $(\operatorname{Rec})$, depredadores (D). Se determinó la biomasa individual promedio y su variación para invertebrados asociados a macrófitas (Tabla 1). Para algunos taxones se midió la biomasa en peso húmedo o peso seco de los invertebrados en balanza OHAUS de 0,01 mg de precisión mediante la técnica empleada por Bonomi (1962). En el caso de los pesajes en seco, se obtuvo luego de secado en estufa a $60^{\circ} \mathrm{C}$ hasta peso constante. Cuando fue posible, se obtuvo la biomasa en peso seco empleando factores de corrección, peso seco/peso húmedo o peso seco/longitud o diámetro, disponibles para los mismos grupos en la región (Zilli et al., 2017). La longitud total o el diámetro mayor se midieron en lupa estereoscópica con escala milimétrica y en microscopio óptico con escala micrométrica.

\section{Análisis numéricos y estadísticos de datos}

Como atributos de los ensambles pleustónicos se determinaron la biomasa, densidad y riqueza de taxones y grupos funcionales. Se realizaron correlaciones entre los atributos de los ensambles pleustónicos con la biomasa de partes sumergidas de las macrófitas y con la riqueza de plantas por humedal y la complejidad arquitectural de hábitats generada por las partes sumergidas. Se calcularon dos medidas de complejidad:

Bioms $=\sum$ Biomasa de cada especie $* D$ Dsum $=\sum D$ de las especies en cada muestra de macrófitas

La biomasa, densidad y abundancia de invertebrados se transformaron con $\log _{10}(x+1)$. Se evaluaron la homogeneidad de varianza (test de Levene, $a=0,05$ ), independencia (gráficos de dispersión) y normalidad (test de ShapiroWilk, $a=0,05$ ) de los residuos de las regresiones lineales, cumpliéndose los supuestos en cada caso. Se estimaron correlaciones lineales de Pearson y su nivel de significancia $(a=0,05)$ entre la biomasa, densidad, abundancia y riqueza de invertebrados y grupos funcionales con las medidas de complejidad de las muestras de macrófitas. Para el caso de los datos de biomasa de invertebrados, se trabajó con los datos procedentes de 24 muestras de macrófitas, mientras que para la densidad con los de 30 muestras de invertebrados. Todos los análisis fueron realizados con el programa estadístico Past v 3.1.

\section{RESULTADOS}

Se registró una riqueza total de diez taxones de macrófitas: Nymphoides indica (L.) Kuntze, C. caroliniana, P. crassipes, Paspalum repens Bergius, M. aquaticum, Limnobium laevigatum (Humb.\& Bonpl. Ex Willd.) Heine, L. peploides, 
Tabla 1. Invertebrados registrados en raíces y partes sumergidas de las macrófitas indicando grupo taxonómico (GT), Grupo Funcional Trófico (GFT) (RTH: Raspadores, trituradores y herbívoros, Rec: recolectores, D: depredadores) y estado de desarrollo (L: larva; A: adulto).

Table 1. Invertebrates found in roots and submerged parts of the macrophytes. The taxonomic group (GT), the trophic functional group (GFT) are indicated (RTH: scrapers shredders and herbivores, Rec: collectors, D:predators) and stage (L: larva; A: adult).

\begin{tabular}{|c|c|c|c|c|c|}
\hline GT & Taxa & GFT & GT & Taxa & GFT \\
\hline Turbellaria & Indeterminado & $\mathrm{D}$ & Collembola & Indeterminado & $\operatorname{Rec}$ \\
\hline Nematoda & Indeterminado & D & Paleoptera & Ephemeroptera & $\operatorname{Rec}$ \\
\hline \multirow[t]{13}{*}{ Oligochaeta } & Allonais inequalis & $\operatorname{Rec}$ & & Anisoptera & D \\
\hline & Stylaria fossularis & $\operatorname{Rec}$ & & Zygoptera & D \\
\hline & Slavina evelinae & $\operatorname{Rec}$ & Hemiptera & Belostomatidae & D \\
\hline & Dero (Aulophorus) furcatus & $\operatorname{Rec}$ & & Pleidae & D \\
\hline & Pristina leidyi & $\operatorname{Rec}$ & & Veliidae & D \\
\hline & P. biserrata & $\operatorname{Rec}$ & & Mesoveliidae & D \\
\hline & P. macrochaeta & $\operatorname{Rec}$ & & Naucoridae & D \\
\hline & P. proboscidea & $\operatorname{Rec}$ & Coleoptera & Noteridae (A y $L$ ) & D \\
\hline & Dero (Dero) multibranchiata & $\operatorname{Rec}$ & & Dytiscidae (A y L) & D \\
\hline & D. (D.) nivea & $\operatorname{Rec}$ & & Gyrinidae (A) & D \\
\hline & D. (D.) obtusa & $\operatorname{Rec}$ & & Elmidae (A y L) & $\operatorname{Rec}$ \\
\hline & D. (D.) botrytis & $\operatorname{Rec}$ & & Hydrophilidae (L y A) & D-Rec \\
\hline & D. (D.) sawayai & $\operatorname{Rec}$ & & Curculionidae (A y L) & RTH \\
\hline Hirudinea & Indeterminado & D & & Staphylinidae (A) & RTH \\
\hline \multirow[t]{8}{*}{ Gastropoda } & Limnoperna fortunei & $\operatorname{Rec}$ & Trichoptera & Indeterminado (L) & $\operatorname{Rec}$ \\
\hline & Sphaeriidae-Euperinae & $\operatorname{Rec}$ & Diptera & Tanypodinae (L) & $\mathrm{D}$ \\
\hline & Eupera sp. & $\operatorname{Rec}$ & & Stratiomyidae (L) & $\operatorname{Rec}$ \\
\hline & Asolene sp. & RTH & & Mucidae (L) spp. & $D$ \\
\hline & Planorbidae & RTH & & Chironominae $(\mathrm{L})$ & $\operatorname{Rec}$ \\
\hline & Heleobia spp. & RTH & & Psychodidae (L) & $\operatorname{Rec}$ \\
\hline & Succineidae & RTH & Lepidoptera & Crambidae (L) & RTH \\
\hline & Ancylidae & RTH & & Phycitinae (L) & RTH \\
\hline \multirow[t]{3}{*}{ Crustacea } & Macrobrachium borellii & RTH & & Noctuidae (L) & RTH \\
\hline & Palaemonetes argentinus & D & Acari & Hidracarina & $\mathrm{D}$ \\
\hline & Hyalella curvispina & RTH & & & \\
\hline
\end{tabular}

P. stratiotes, Hydrocotyle ranunculoides L. y Salvinia spp. La mayor abundancia de partes sumergidas fue registrada para $P$. crassipes, luego $L$. peploides, N. indica y $P$. repens (Figura 2a). La biomasa promedio de macrófitas fue de

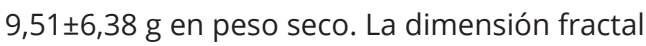
osciló entre 1,17 (Salvinia spp.) y 1.57 ( N. indica) (Figura 2b).

La riqueza de invertebrados fue de 53 taxones (Tabla 1). El grupo RTH tuvo la mayor biomasa y los recolectores la mayor densidad (Figura 3). La biomasa promedio de invertebrados fue de 29,51 $\pm 145,10 \mathrm{~g}$ ind/g en peso seco de macrófitas. Gastropoda fue dominante en biomasa, seguido por Coleoptera y Diptera (Tabla 2), (Figura 4). La densidad promedio de invertebrados fue de $25 \pm 92 \mathrm{ind} / \mathrm{g}$ en peso seco de macrófitas. Crustacea dominó en densidad, luego Gastropoda y Diptera. Entre los crustáceos dominaron los microcrustáceos (copépodos, cladóceros, ostrácodos), principalmente planctónicos. Dado que no se estimó su biomasa, éstos no fueron considerados en los análisis posteriores.

La biomasa, riqueza y diversidad total de invertebrados no presentaron correlaciones significativas con la complejidad de macrófitas (correlación de Pearson, p>0,05). La densidad total se correlacionó significativa y negativamente con la complejidad de macrófitas 'Bioms' $(p<0,01)$. La densidad y riqueza de RTH aumentaron significativamente con la riqueza de macrófitas $(p<0,02)$ y su complejidad fractal 'Dsum' $(p<0,02)$, mientras que la densidad de 'Rec' disminuyó con la complejidad 'Bioms'. No se registraron correlaciones significativas de los atributos para los depredadores con la complejidad generada por las macrófitas.

\section{DISCUSIÓN}

La medición de la dimensión fractal (D) calculadas al parecer fueron buenos indicadores de complejidad estructural, a pesar de que 


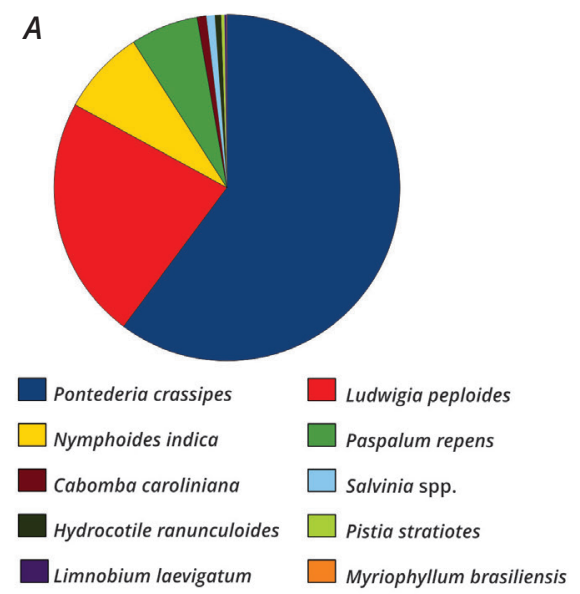

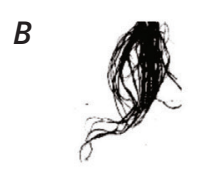

Nymphoides indica $D=1,57 \pm 0,04$

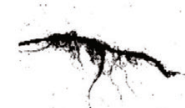

Myriophyllum acuaticum Lim $D=1,48 \pm 0,04$

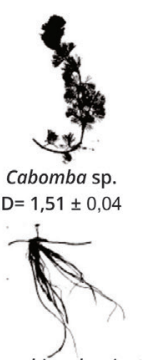

$\mathrm{D}=1,46 \pm 0,05$

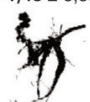

Hydrocotyle sp. $\mathrm{D}=1,28 \pm 0,05$
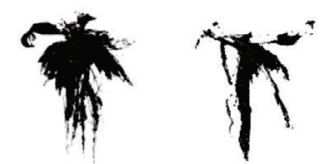

Pontederia crassipes Paspalum repens $D=1,48 \pm 0,05 \quad D=1,48 \pm 0,05$
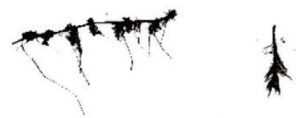

Pistia stratiotes

Figura 2 a. Representación porcentual de la biomasa de las partes sumergidas de las especies de macrófitas en las lagunas, b. imágenes de raíces y partes sumergidas de las macrófitas a las que se le determino la dimensión fractal promedio (D).

Figure 2 a. Porcentual representation of submerged portions of macrophyte species in lakes, $\mathbf{b}$. images of roots and submerged parts of macrophytes for which the average fractal dimension was determined (D).

no se encontró relación entre la biomasa, riqueza y diversidad de invertebrados con la complejidad generada por las macrófitas. A partir de los resultados, se pudo observar que las macrófitas que exhibieron mayor número raíces y partes sumergidas y a su vez formas más finamente divididas tuvieron mayor valor $D$

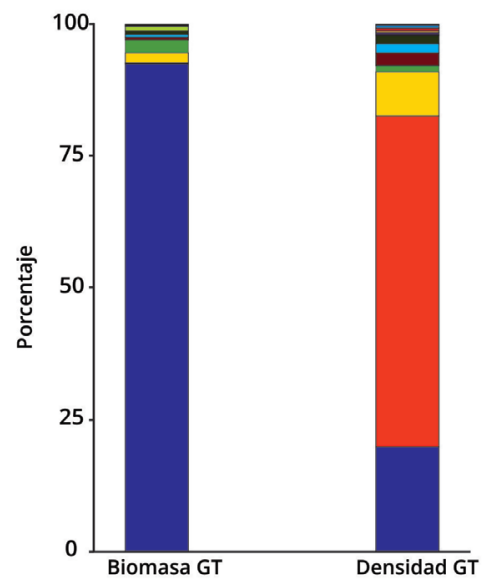

\section{$\square$ Gastropoda $\square$ Crustacea $\square$ Diptera $\square$ Coleoptera $\square$ Oligochaeta $\square$ Hirudinea $\square$ Paleoptera $\square$ Turbellaria $\square$ Hemiptera $\square$ Lepidoptera $\square$ Nematoda $\square$ Acari - Trichoptera}

Figura 3. Biomasa y densidad porcentual de grupos funcionales tróficos.

Figure 3. Biomass and relative density of functional feeding groups.
(N. indica y C. caroliniana). Las macrófitas menos complejas (valores de D más bajo) parecen ser aquellas con formas más simples y lineales (Salvinia sp.). En este estudio encontramos que las macrófitas en humedales de llanura del río Paraná sostienen una alta diversidad, densidad y biomasa de taxones y de diferentes grupos

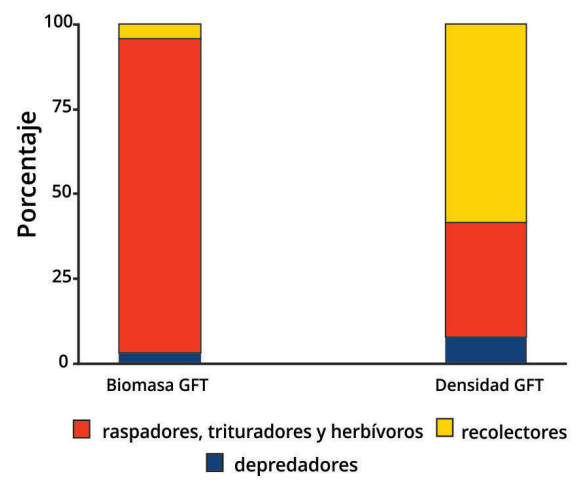

Figura 4. Biomasa y densidad porcentual de los grupos taxonómicos de invertebrados pleustónicos. GT: grupos taxonómicos.

Figure 4. Biomass and relative density of the taxonomic groups of pleuston. GT: taxonomic groups. 
Tabla 2. Promedio y desvío estándar de la biomasa individual en peso húmedo (X் Bh \pm Bh) y peso seco (X் Bs $\pm \mathrm{Bs}$ ) de los taxones pleustónicos registrados (Be: biomasa en peso seco estimada empleando factores de corrección a partir de la relación biomasa seca/biomasa húmeda; M: biomasa en peso seco a partir de relaciones morfométricas biomasa/longitud o diámetro).

Table 2. Mean and standard deviation of wet biomass ( $\dot{\mathrm{X}} \mathrm{Bh} \pm \mathrm{Bh}$ ) and dry biomass ( $\dot{\mathrm{X}} \mathrm{Bs} \pm \mathrm{Bs})$ of recorded pleustonic taxa (Be: dry biomass estimated by using dry mass/wet mass correction factors; M: dry biomass estimated by using dry weight correction factors/body length or diameter).

\begin{tabular}{|c|c|c|c|c|c|c|c|c|}
\hline \multirow[t]{2}{*}{ Taxones } & \multirow[t]{2}{*}{$\dot{\mathrm{x}} \mathrm{Bh}$} & \multirow[t]{2}{*}{$\pm \mathrm{Bh}$} & \multirow[t]{2}{*}{$\dot{x} B s$} & \multirow[t]{2}{*}{ \pm Bs } & \multicolumn{4}{|c|}{ Método } \\
\hline & & & & & $\mathbf{B h}$ & Bs & $\mathrm{Be}$ & M \\
\hline Turbellaria & 0,029 & 0,072 & 0,023 & 0,022 & $x$ & $\mathbf{x}$ & & \\
\hline Hirudinea & 0,020 & 0,034 & 0,011 & 0,014 & $x$ & $\mathbf{x}$ & & \\
\hline Dero (Dero) nivea & - & - & 0,0005 & 0,0008 & & & & $x$ \\
\hline Pristina leidyi & - & - & 0,00006 & 0,0002 & & & & $x$ \\
\hline Allonais inaequalis & - & - & 0,0006 & 0,001 & & & & $x$ \\
\hline Stylaria fossularis & - & - & 0,00009 & 0,0003 & & & & $x$ \\
\hline Pristina macrochaeta & - & - & 0,0001 & 0,0003 & & & & $x$ \\
\hline Pristina proboscidea & - & - & 0,00003 & 0,0002 & & & & $x$ \\
\hline Dero (Dero) & & & & & & & & \\
\hline multibranchia & - & - & 0,0002 & 0,0007 & & & & $\mathbf{x}$ \\
\hline Pristina biserrata & - & - & 0,00002 & 0,0001 & & & & $\mathbf{x}$ \\
\hline Dero (Dero) obtusa & - & - & 0,001 & 0,0007 & & & & $\mathrm{x}$ \\
\hline Dero (Dero) botrytis & - & - & 0,002 & 0,002 & & & & $\mathbf{x}$ \\
\hline Dero (Dero) sawayai & - & - & 0,0005 & 0,0001 & & & & $\mathrm{x}$ \\
\hline Hyalella sp, & 0,002 & 0,006 & 0,0009 & 0,002 & $x$ & $\mathrm{x}$ & & \\
\hline Macrobrachium borellii & 0,241 & 0,195 & 0,044 & 0,036 & $x$ & $\mathrm{x}$ & & \\
\hline Ephemeroptera & 0,002 & 0,002 & 0,006 & 0,021 & $x$ & & & $\mathbf{x}$ \\
\hline Anisoptera & 0,080 & 0,043 & 0,040 & 0,022 & $x$ & & $x$ & \\
\hline Zygoptera & 0,053 & 0,111 & 0,026 & 0,050 & $x$ & & $\mathbf{x}$ & \\
\hline Belostomatidae & 0,014 & 0,009 & 0,007 & 0,004 & $\mathbf{x}$ & $\mathbf{x}$ & & \\
\hline Pleidae & 0,011 & 0,013 & 0,009 & 0,007 & $\mathbf{x}$ & $\mathbf{x}$ & & \\
\hline Noteridae (A) & 0,118 & - & - & - & $x$ & & $\mathbf{x}$ & \\
\hline Noteridae (L) & 0,001 & 0,0005 & 0,0003 & 0,0003 & $\mathbf{x}$ & & $\mathbf{x}$ & \\
\hline Dytiscidae (A) & 0,065 & 0,112 & 0,033 & 0,056 & $\mathbf{x}$ & & $\mathbf{x}$ & \\
\hline Dytiscidae (L) & 0,010 & 0,014 & 0,00009 & 0,0004 & $x$ & & $\mathbf{x}$ & \\
\hline Curculionidae (A) & 0,059 & 0,082 & 0,029 & 0,041 & $x$ & & $\mathrm{x}$ & \\
\hline Curculionidae (L) & 0,0006 & 0,0005 & - & - & $\mathrm{x}$ & & & \\
\hline Elmidae (A) & 0,115 & 0,163 & 0,058 & 0,081 & $x$ & & $x$ & \\
\hline Hydrophilinae (A) & 0,013 & - & - & - & $x$ & & & \\
\hline Hydrophilinae (L) & 0,111 & - & - & - & $x$ & & & \\
\hline Stratiomyidae (L) & - & - & 0,0005 & 0,001 & & & & $\mathbf{x}$ \\
\hline Crambidae (L) & 0,737 & 1,042 & 0,035 & 0,049 & $x$ & & & $\mathbf{x}$ \\
\hline Trichoptera (L) & 0,025 & - & 0,0001 & - & $\mathbf{x}$ & & & $\mathbf{x}$ \\
\hline Chironominae (L) & - & - & 0,0002 & 0,0002 & & & & $\mathbf{x}$ \\
\hline Tanypodinae (L) & - & - & 0,0005 & 0,0007 & & & & $\mathbf{x}$ \\
\hline Muscidae (L) & - & - & 0,0003 & 0,0008 & & & & $\mathrm{x}$ \\
\hline Succineidae & 0,013 & 0,021 & 0,005 & 0,008 & $\mathrm{x}$ & $\mathbf{x}$ & & \\
\hline Ancylidae & 0,009 & 0,017 & 0,011 & 0,017 & $x$ & $\mathbf{x}$ & & \\
\hline Planorbidae & - & - & 0,140 & 0,407 & & & & $\mathrm{x}$ \\
\hline Heleobia sp, & - & - & 0,030 & 0,050 & & & & $\mathrm{x}$ \\
\hline Sphaeriidae & - & - & 0,121 & 0,047 & & & & $\mathbf{x}$ \\
\hline Limnoperna fortunei & & & 0,304 & 0,261 & & & & $\mathbf{x}$ \\
\hline
\end{tabular}

funcionales de macroinvertebrados. Estos resultados coinciden con lo encontrado en otros estudios en grandes ríos (Blanco-Belmonte et al., 1998; Poi de Neiff, 2003). La biomasa estimada para los macroinvertebrados fue cinco veces superior a la de los invertebrados bentónicos en lagunas de la llanura aluvial en la misma región (Zilli, 2013) y casi 145 veces superior a la comunidad bentónica en los grandes cauces del río Paraná (Marchese, 1983). Al igual que en estos estudios, como así en otros para el pleuston de la región (Paporello de Amsler, 1983, 1987 a, b) y del río Paraguay (Poi de Neiff, 2003), la composición funcional estuvo dominada por los recolectores y raspadores. Si se considera que la biomasa de macrófitas en humedales aluviales puede ser de entre 4,18 y 59,04 ton en peso húmedo/ ha (Sabattini \& Lallana, 2007) y que éstas son generadoras de hábitats para una diversa, abundante y productiva biota acuática, podríamos inferir que las comunidades pleustónicos representan 
una fuente muy importante de propágulos y energía para el sistema. Proveerían entonces una alta proporción de la producción secundaria dentro de los ambientes acuáticos en el interior de la llanura aluvial. Frecuentemente derivan masas de macrófitas desde la llanura hacia el río debido a la acción del viento e inundaciones (Sabattini \& Lallana, 2007) y se ha demostrado que en grandes ríos esta deriva es importante para animales (Bulla et al., 2011; Poi de Neiff \& Zozaya, 1989; Schiesari et al., 2003) y propágulos vegetales producidos dentro de la llanura aluvial (Facelli et al., 2019), con efectos incluso a escala biogeográfica (Guerrero et al., 2017). Nuestros resultados demuestran que en las macrófitas habitan diversos grupos de invertebrados, que en su conjunto alcanzan una gran abundancia y que por tanto aportan en gran medida a la alta producción y diversidad acuática (anélidos, moluscos, muchos insectos) y riparia de la llanura (principalmente insectos, pero también moluscos). Asimismo, aportan diferentes funciones a los procesos en los humedales dado que se registraron tanto diferentes taxones de detritívoros, así como de depredadores. Por todo esto se puede concluir que contribuyen a la resiliencia de los ambientes acuáticos locales y posiblemente del sistema.

Por otra parte, se encontraron evidencias para no aceptar la hipótesis planteada. Si bien una alta riqueza, densidad y biomasa de invertebrados y de la proporción de raspadores y depredadores están a menudo correlacionadas con una vegetación acuática diversificada y abundante (Diehl, 1992; Crowder \& Cooper, 1982; Gilinsky, 1984) con mayor riqueza de pleuston en especies vegetales de mayor biomasa (Poi de Neiff y Neiff, 2006). En nuestro estudio no se registraron correlaciones con la mayoría de los atributos para todo el ensamble, mientras que la densidad total y la de recolectores se correlacionaron significativa y negativamente con la complejidad (Bioms). Estas tendencias, podrían explicarse más por factores definidos por variables no consideradas en nuestro estudio vinculadas a la conectividad de las lagunas, su ubicación dentro de la llanura y su distancia al cauce principal que afectarían la distribución de los taxones menos específicos de los sustratos vegetales. Para el bentos por ejemplo existe un incremento de la diversidad y biomasa desde el cauce principal hacia los ambientes en el interior de la llanura aluvial (Ezcurra de Drago et al., 2007), mientras que la biomasa, diversidad y densidad de diferentes grupos taxonómicos y funcionales de macroinvertebrados se vincula principalmente a la conectividad de las lagunas y tipos de ambiente (Drago et al., 2003; Zilli, 2013; Zilli \& Marchese, 2011). La uniformidad en la abundancia de presas en todas las muestras, tanto de macroinvertebrados como de microcrustáceos registrada en nuestro estudio que también ha sido reportada ampliamente en la bibliografía para la región (José de Paggi y Paggi, 2007; Poi de Neiff y Neiff, 2006) puede haber enmascarado el efecto de la complejidad vegetal sobre la abundancia de depredadores. Se encontraron evidencias para apoyar la predicción vinculada a la relación entre la complejidad del sustrato con los taxones de mayor especificidad (trituradores, herbívoros, raspadores), al menos en densidad y riqueza. La ausencia de relación entre biomasa de este grupo con la complejidad vegetal puede vincularse a la abundancia uniforme de gastrópodos que tuvieron una elevada biomasa individual, así como a nivel del ensamble en general. La vinculación de la riqueza y densidad de invertebrados con la arquitectura vegetal puede relacionarse con las variaciones que el conjunto de estas características genera en los microhábitats (variaciones en la penetración lumínica, retención de sedimentos, circulación de agua y nutrientes, etc.) que a su vez impactan en el desarrollo del perifiton (Neiff \& Poi de Neiff, 2006). Por otra parte, si bien estudios realizados en humedales de la llanura aluvial aguas arriba del sitio del presente estudio, encontraron que la riqueza de especies de invertebrados variaba entre especies de macrófitas y estaba en relación con la biomasa de las plantas en stands monoespecíficos (Poi de Neiff y Neiff, 2006), otros estudios demostraron la afinidad de los invertebrados por ciertas especies de plantas en relación con la arquitectura vegetal en stands pluriespecíficos (Poi de Neiff y Neiff, 1984). Esto podría explicar la ausencia de correlación de los atributos de la comunidad con la biomasa de partes sumergidas y la correlación positiva con la complejidad fractal y riqueza de macrófitas. En conclusión, las macrófitas son generadoras de hábitat para diversos y abundantes 
macroinvertebrados en el río Paraná, vinculándose sus atributos a la complejidad de las partes sumergidas diferencialmente de acuerdo con su especificidad por el sustrato. La gran biomasa vegetal en ambientes de la llanura podría sostener la mayor diversidad y producción de invertebrados de los ambientes al comparar con otros ensambles (ej. bentos). Las macrófitas y los invertebrados pleustónicos intervienen en diferentes procesos locales y regionales por deriva, por lo que aportarían a la resiliencia del sistema río-llanura aluvial. La información aquí obtenida puede ser útil para el desarrollo de estrategias de manejo y remediación de humedales.

\section{AGRADECIMIENTOS}

Agradecemos a la Dra. Mercedes R. Marchese por la asistencia en la identificación de oligoquetos. Este estudio fue posible gracias al Proyecto PICT 2012-2791 Agencia Nacional de Investigaciones Científicas y Técnicas (ANPCyT, MinCyT) y al Proyecto PIP 318 Consejo Nacional de Investigaciones Científicas y Técnicas (CONICET), Argentina. Agradecemos a los dos revisores anónimos ya que sus sugerencias enriquecieron este trabajo.

\section{REFERENCIAS}

Arrington, D. A., Winemiller, K. O. \& Layman, C. A. (2005). Community assembly at the patch scale in a species rich tropical river. Oecologia, 144: 157-167.

Agostinho, A. A, Gomes, L. C y Julio, H. F. (2003). Relacões entre macrófitas e fauna de peixes. En Thomaz, S. M. \& Bini, L. M. (Eds.). Ecologia e manejo de macrófitas aquáticas (pp. 261-276) Maringá: Eduem.

Blanco-Belmonte, L., Neiff, J. J. \& Poi de Neiff, A. (1998). Invertebrate fauna associated with floating macrophytes in the floodplain lakes of the Orinoco (Venezuela) and Paraná (Argentina). Verhandlungen des Internationalen Verein Limnologie, 26: 2030-2034.
Bonomi, G. (1962). La dinámica produttiva delle prinipali propolacioni macrobentonice del Lago di Varece. Memorie dell'Instituto Italiano di Idrobiologia, 15: 207-254.

Brinkhurst, R. O. y Marchese, M. R. (1989). Guía para la identificación de Oligoquetos acuáticos continentales de Sud y Centroamérica. Colección Climax. Santa Fe: Asociación Ciencias Naturales del Litoral.

Brown, J. H., Gupta, V. K., Li. B-L., Milne, B. T., Restrepo, C. \& West, G. B. (2002). The fractal nature of nature: power laws, ecological complexity and biodiversity. Philosophical transactions of the Royal Society of London Series $B$, Biological sciences, 357: 619-626.

Bulla, C. K., Gomes, L. C., Miranda, L. E. \& Agostinho, A. A. (2011). The ichthyofauna of drifting macrophyte mats in the Ivinhema River, upper Parana River basin, Brazil. Neotropical Ichthyology, 9: 403-409.

Burkart, A. (1957). Ojeada sinóptica sobre la vegetación del Delta del río Paraná. Darwiniana, 11: 457-561

Burkart, A. (Dir.). (1969). Flora llustrada de Entre Ríos (Argentina). Gramíneas. Buenos Aires: Instituto Nacional de Tecnología Agropecuaria.

Burkart, A. E. (Dir.). (1974). Flora ilustrada de Entre Ríos (Argentina). Tomo 6 Parte VI: Dicotiledóneas Metaclamídeas (Gamopétalas). Rubiales, Cucurbitales, Campanulales. Buenos Aires: Instituto Nacional de Tecnología Agropecuaria.

Burkart, A. y Bacigalupo, N. M. (Dirs.). (1979). Flora ilustrada de Entre Ríos (Argentina). Tomo 5. Dicotiledoneas Metaclamideas Generalidades; (Gamopétalas) A: Primulales, Plumbaginales, Ebenales, Contortales, Tubiflorales, Callitrichales, Plantaginales. Buenos Aires: Instituto Nacional de Tecnología Agropecuaria.

Burkart, R., Bárbaro, N. O., Sánchez, R. O., \& Gómez, D. A. (1999). Eco-regiones de la Argentina. Buenos Aires: Administración de Parques Nacionales.

Cabrera, A. L. (Dir.). (1968). Flora de la Provincia de Buenos Aires: I Pteridófitas-Gimnospermas 
y Monocotiledóneas (excepto Gramíneas). Colección Científica. Buenos Aires: Instituto Nacional de Tecnología Agropecuaria.

Cheruvelil, K. S., Soranno, P. A., Madsen, J. D. \& Roberson, M. J. (2002). Plant architecture and epiphytic macroinvertebrate communities: the role of an exotic dissected macrophyte. Journal of the North American Benthological Society, 21: 261-277.

Crowder, L. B. \& Cooper, W. E. (1982). Habitat structural complexity and the interaction between bluegills and their prey. Ecology, 63:1802-1813.

Cummins, K. W., Merritt, R. W. \& Andrade, P. C. N. (2005). The use of invertebrate functional groups to characterize ecosystem attributes in selected streams and rivers in south Brazil. Studies on Neotropical Fauna and Environment, 40: 69-89.

Cyr, H. \& Downing, J. A. (1988). The abundance of phytophilous invertebrates on different species of submerged macrophytes. Freshwater Biology, 20: 365-374.

Dibble, E., \& Thomaz, S. (2009). Use of fractal dimension to assess habitat complexity and its influence on dominant invertebrates inhabiting tropical and temperate macrophytes. Journal of Freshwater Ecology, 24, 93-102.

Diehl, S. (1992). Fish predation and benthic community structure: the role of omnivory and habitat complexity. Ecology, 73: 1646-1661.

Domínguez, E. y Fernández, H. R. (2009). Macroinvertebrados bentónicos sudamericanos: sistemática y biología. Tucumán: Fundación Miguel Lillo.

Drago E. C., Ezcurra de Drago I., Oliveros O. B. \& Paira A. R. (2003). Aquatic habitats, fish and invertebrate assemblages of the Middle Paraná River. Amazoniana, 17: 291-341.

Dvořak, J. \& Best, E. P. H. (1982). Macroinvertebrate communities associated with the macrophytes of Lake Vechten- structural and functional relationships. Hydrobiologia, 95: 115-126.

Ezcurra de Drago, E. C., Marchese, M. R. \& Montalto, L. (2007). Benthic Invertebrates. In:
Iriondo, M., Paggi, J. C. \& Parma, M. J. (Eds.). The Middle Paraná River: Limnology of a Subtropical Wetland (pp. 83-122). Berlin Heidelberg New York: Springer.

Facelli, F., Schneider, B. \& Zilli, F. (2019). Factors driving seed dispersal in a Neotropical riverfloodplain system. Acta Botanica Brasilica (in press).

Feldman, R. S. (2001). Taxonomic and size structures of phytophilous macroinvertebrate communities in Vallisneria and Trapa beds of the Hudson River, New York. Hydrobiologia, 452: 233-245.

Franceschi, E. A., Prado, D. E. y Lewis, J. P. (1985). Comunidades vegetales y mapa de vegetación de la reserva "El Rico" e islas aledañas (Provincia de Santa Fe, República Argentina). Rosario: Universidad Nacional de Rosario.

Galizzi, C., Zilli, F. L. \& Marchese, M. R. (2012). Diet and functional feeding groups of Chironomidae (Diptera) in the Middle Paraná River floodplain (Argentina). Iheringia Série Zoologia, 102: 117 121.

Gilinsky, E. (1984). The role of fish predation and spatial heterogeneity in determining benthic community structure. Ecology, 65: 455-468.

Giorgi A. D. N., Feijoó, C. S. \& Tell, G. (2005). Primary producers in a Pampean stream: temporal variation and structuring role. Biodiversity and Conservation, 14: 1699-1718.

Guerrero, E. L., Agnolin, F. L., Grilli, P., Suazo Lara, F. A., Boné, E., Tenorio, A B., Derguy, M., Lucero, S., Chimento Ortíz, N. R., Milat, J. A., Nenda, S., Benedicto, M., Montalibet, E. Olmos, M., Barrasso, D. y Apodaca, M. J. (2017). Inventario de la fauna transportada por balsas de vegetación flotante en el Sistema fluvial del Río de La Plata. Revista del Museo Argentino de Ciencias Naturales n.s., 19: 177-183.

Hanson, J. M. (1990). Macroinvertebrate sizedistributions of two contrasting freshwater macrophyte communities. Freshwater Biology, 24: 481-491.

Higuti, J., Velho, F. M., Lansac-Toha, F. A. \& Martens, K. (2007). Pleuston communities are buffered from regional flood pulses: The example of ostracods in the Parana River 
floodplain, Brazil. Freshwater Biology, 52: 19301943.

José de Paggi, S. \& Paggi, J. C. (2007). Zooplankton. In: Iriondo, M., Paggi, J. C. \& Parma, M. J. (Eds.). The Middle Paraná River: Limnology of a Subtropical Wetland (pp. 83-122). Berlin Heidelberg New York: Springer.

Kelaher, B. P. (2003). Changes in habitat complexity negatively affect diverse gastropod assemblages in coralline algal turf. Oecologia, 135: 431-441.

Li, B. L. (2000). Fractal geometry applications in description and analysis of patch patterns and patch dynamics. Ecological Modelling, 132: 33-50.

MacArthur, R. H. \& MacArthur, J. W. (1961). On bird species diversity. Ecology, 42: 594-598.

Marchese, M. R. (1983). Population dynamics of Narapa bonettoi Righi and Varela, (Oligochaeta: Narapidae) from the main channel of the Middle Paraná River, Argentina. Hydrobiologia, 278: 303-308.

McAbendroth, L., Ramsay, P. M., Foggo, A., Rundle, S. D. \& Bilton, D. T. (2005). Does macrophyte fractal complexity drive invertebrate diversity, biomass and body size distributions? Oikos, 111: $279-290$.

Merritt, R. W. \& Cummins, K. W. (1996). An introduction to the aquatic insects or North America. 3rd.ed. Dubuque, lowa: Kendall Hunt. Publishing Company.

Mormul, R. P., Thomaz, S. M., Takeda, A. M. \& Behrend, R. D. (2011). Structural complexity and distance from source habitat determine invertebrate abundance and diversity. Biotropica, 43: 738-745.

Neiff, J.J. \& Poi de Neiff, A. (2003). Connectivity processes as a basis for management of aquatic plants. In: Thomaz, S. M. \& Bini, M. L. (Eds.). Ecologia e manejo de macrófitas aquáticas (pp. 39-58). Maringá: Eduem.

Neiff, J. J. y Poi de Neiff, A. (2006). Riqueza de especies y similaridad de los invertebrados que viven en plantas flotantes de la planicie de inundación del rio Paraná (Argentina). Interciencia, 31: 220-225.
Neiff, J. J., Casco, S. L., Mari, E. C. K., Di Rienzo, J. A., \& Poi, A. S. G. (2014). Do aquatic plant assemblages in the Paraná River change along the river's length? Aquatic Botany, 114: 50-57.

O'Hare, M. T., Aguiar, F. C., Asaeda, T., Bakker, E. S., Chambers, P. A., Clayton, J. S., Elger, A., Ferreira, T. M., Gross, E. M., Gunn, I. D. M. \& Gurnell, A. M. (2018). Plants in aquatic ecosystems: current trends and future directions. Hydrobiologia, 812: 1-11.

Padial, A. A., Thomaz, S. M. \& Agostinho, A. A. (2009). Effects of structural heterogeneity provided by the floating macrophyte Eichhornia azurea on the predation efficiency and habitat use of the small Neotropical fish Moenkhausia sanctaefilomenae. Hydrobiologia, 624:161-170.

Paporello de Amsler, G. (1983). Fauna asociada a las raíces de Eichhornia crassipes en el río Correntoso (Prov. de Santa Fe): Estudio Preliminar. Natura Neotropicalis, 2:133-147.

Paporello de Amsler, G. (1987a). Fauna asociada a las raíces de Eichhornia crassipes en cauces secundarios y tributarios del río Paraná en el tramo Goya-Diamante. Natura Neotropicalis, $1: 37-50$.

Paporello de Amsler, G. (1987b). Fauna asociada a las raíces de Eichhornia crassipes en una laguna del valle aluvial del río Paraná (Los Matadores, Santa Fe, Argentina). Natura Neotropicalis, 1:93-103.

Poi de Neiff, A. (2003). Macroinvertebrates living on Eichhornia azurea Kunth in the Paraguay River. Acta Limnologica Brasiliensia, 15: 55-63.

Poi de Neiff, A. y Neiff, J. J. (1984). Dinámica de la vegetación acuática y su fauna. Physis, 42:53-67.

Poi de Neiff, A. y Neiff, J. J. (2006). Riqueza de especies y similaridad de los invertebrados que viven en plantas flotantes de la planicie de inundación del río Paraná (Argentina). Interciencia, 31: 220-225.

Poi de Neiff, A. y Zozaya, I. Y. B. (1989). Efectos de las crecidas sobre las poblaciones de invertebrados que habitan macrofitas emergentes en el río Paraná. Revue d'Hydrobiologie tropical, 22: 13-20. 
Pott, V. J. \& Pott, A. (2000). Plantas aquáticas do Pantanal. Brasilia: EMBRAPA Comunicação para transferência de Tecnologia.

Ricklefs, R. E. \& Lovette, I. J. (1999). The roles of island area per se and habitat diversity in the species-area relationships of our lesser Antillean faunal groups. Journal of Animal Ecology, 68: 1142-1160.

Sabattini, R. A. \& Lallana, V. H. (2007). Aquatic macrophytes. In: Iriondo M., Paggi, J. C y Parma, M. J. (Eds.). The Middle Paraná River Limnology of a Subtropical Wetland (pp. 205-226). Berlin Heidelberg, New York: Springer-Verlag.

Sand-Jensen, K. (1998). Influence of submerged macrophytes on sediment composition and near-bed flow in lowland streams. Freshwater Biology, 39: 663-679.

Saravia, L. A., Giorgi, A. \& Momo, F. (2012). Multifractal spatial patterns and diversity in an ecological succession. PloS one, 7:e34096.

Schiesari, L. Z., Azevedo-Ramos, J., Garcia, C., Gordo, M., Messias, M., \& Monteiro Vieira, E. (2003). Macrophyte rafts as dispersal vectors for fishes and amphibians in the Lower Solimes River, Central Amazon. Journal of Tropical Ecology, 19: 333-336.

Schneider, B., Cunha, E. R., Marchese, M. \& Thomaz, S. M. (2015). Explanatory variables associated with diversity and composition of aquatic macrophytes in a large subtropical river floodplain. Aquatic Botany, 121: 67-75.
Souza Franco, G. M. \& Takeda, A. M. (2000). Invertebrates associated to Paspalum repens (Poacea) at the mouth of Caracu stream (19911992), affluent of the Paraná River, Porto Rico (PR), Brazil. Brazilian Archives of Biodiversity and Technology, 43: 317-325.

Thomaz, S. M., Dibble, E. D., Evangelista, L. R., Higuti, J., \& Bini, L. M. (2008). Influence of aquatic macrophyte habitat complexity on invertebrate abundance and richness in tropical lagoons. Freshwater Biology, 53: 358-367.

Zhang, Y., Jeppesen, E., Liu, X., Qin, B., Shi, K., Zhou, Y., Thomaz, M. T. \& Deng, J. (2017). Global loss of aquatic vegetation in lakes. Earth-Science Reviews, 173: 259-265.

Zilli, F. (2013). Distribution of benthic invertebrate biomass and secondary production in relation to floodplain connectivity in a large river system (Paraná River, Argentina). International Review of Hydrobiology, 98: 284-293.

Zilli, F. L. \& Marchese, M. R. (2011). Patterns in macroinvertebrate assemblages at different spatial scales.Implications of hydrological connectivity in a large floodplain river. Hydrobiologia, 663: 245-257.

Zilli, F. L, Barco del, J. \& Vanzetti, A. (2017). Biometry of neotropical invertebrates inhating floodplain rivers: unraveling bionomy. Iheringia Série Zoologia, 107:e2017014. 